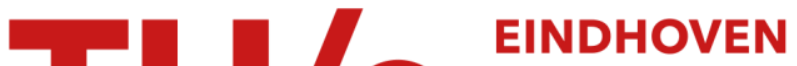 UNIVERSITY OF TECHNOLOGY
}

\section{Vibration isolation of a electromagnetic actuator with passive gravity compensation}

\section{Citation for published version (APA):}

Ding, C., Damen, A. A. H., Bosch, van den, P. P. J., \& Janssen, J. L. G. (2010). Vibration isolation of a electromagnetic actuator with passive gravity compensation. In Proceedings of the 2nd International Conference on Computer and Automation Engineering (ICCAE), 26-28 February 2010, Singapore (pp. 85-89). Institute of Electrical and Electronics Engineers. https://doi.org/10.1109/ICCAE.2010.5451993

DOI:

10.1109/ICCAE.2010.5451993

Document status and date:

Published: 01/01/2010

\section{Document Version:}

Publisher's PDF, also known as Version of Record (includes final page, issue and volume numbers)

\section{Please check the document version of this publication:}

- A submitted manuscript is the version of the article upon submission and before peer-review. There can be important differences between the submitted version and the official published version of record. People interested in the research are advised to contact the author for the final version of the publication, or visit the $\mathrm{DOI}$ to the publisher's website.

- The final author version and the galley proof are versions of the publication after peer review.

- The final published version features the final layout of the paper including the volume, issue and page numbers.

Link to publication

\section{General rights}

Copyright and moral rights for the publications made accessible in the public portal are retained by the authors and/or other copyright owners and it is a condition of accessing publications that users recognise and abide by the legal requirements associated with these rights.

- Users may download and print one copy of any publication from the public portal for the purpose of private study or research.

- You may not further distribute the material or use it for any profit-making activity or commercial gain

- You may freely distribute the URL identifying the publication in the public portal.

If the publication is distributed under the terms of Article $25 \mathrm{fa}$ of the Dutch Copyright Act, indicated by the "Taverne" license above, please follow below link for the End User Agreement:

www.tue.nl/taverne

Take down policy

If you believe that this document breaches copyright please contact us at:

openaccess@tue.nl

providing details and we will investigate your claim. 


\title{
Vibration Isolation of an Electromagnetic Actuatorwith Passive Gravity Compensation
}

\author{
C. Ding †, A.A.H. Damen†, P.P.J. van den Bosch†, and J.L.G. Janssen $\dagger$ \\ $\dagger$ Department of Electrical Engineering, Eindhoven University of Technology. \\ $\dagger$ Eindhoven, the Netherlands. \\ Email: c.ding@tue.nl, a.a.h.damen@tue.nl, p.p.j.v.d.bosch@tue.nl, j.l.g.janssan@tue.nl
}

\begin{abstract}
A control strategy of combining $H_{\infty}$ control and feedback linearization was applied to the model of a highly nonlinear, three Degrees-Of-Freedom (DOF) electromagnetic actuator, which was recently designed for non-contact suspension of a large payload. The new electromagnetic actuator has the advantage of passive gravity compensation based on permanent magnets with low stiffness and high force density. But the nonlinearity is so high that the stability status along each DOF changes while the translator is traveling within the working range. Feedback linearization method was used to compensate the nonlinearity, a stabilizing controller was employed to eliminate the slow-varying calculation error of the passive force, and an $H_{\infty}$ controller was designed for vibration isolation. Simulation results show that the proposed control strategy has robust vibration isolation performance within a working range in which the relation between the magnetic force and the relative position is highly nonlinear.
\end{abstract}

Keywords-Vibration Isolation, Nonlinear Control, NonContact Electromagnetic Suspension.

\section{INTRODUCTION}

In a micro-lithographic machine, referred to as a waferscanner, shown in Fig. 1(a), a well-performing suspension platform is crucial for the high-accuracy (a few tens of nm) positioning applications. The functionality of this suspension platform includes isolating the payload (a complex lens system) from floor vibrations on six Degrees-Of-Freedom (DOF) in a broad frequency band, compensating the payload gravity in scales of thousands of kilograms, and rejecting the force disturbances directly applied to the payload. The current air-bearing suspension system, shown in Fig. 1(b), compensates the gravity by three air bearings and isolates the floor vibration actively by linear actuators. However, the dynamics of the three air bearings are difficult to accurately model or measure. Moreover, vacuum operation is not feasible. Non-contact electromagnetic actuators could be an alternative solution for the future suspension platform.

Passive gravity compensation in such actuators is achieved by permanent magnets while the stabilization and vibration isolation are achieved by electromagnets. The design challenges for these electromagnetic actuators are limited size, large capability of gravity compensation, low power consumption, robust stability, and good vibration isolation performance. Limited size and large gravity compensation require high force density (the ratio of force

978-1-4244-5586-7/10/\$26.00 (C) 2010 IEEE over volume). Good vibration isolation performance and low power consumption prefer low stiffness.

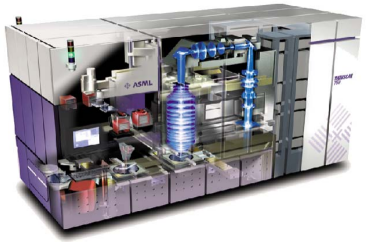

(a) The Wafer-scanner from ASML in Eindhoven, the Netherlands

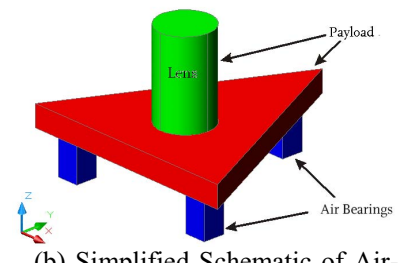

(b) Simplified Schematic of AirBearing Suspension System
Figure 1. A wafer-scanner and its suspension platform

To meet both the force density and the stiffness criterions, a new magnetic topology of a 6-DOF electromagnetic actuator is designed and the passive magnetic force is calculated [3]. A unique relative position exists between the translator and the stator such that the passive gravity compensation force is maximized and all entries of the theoretical stiffness matrix are zero, which is preferred for vibration isolation. However, the relations between the permanent magnetic forces and the three relative Cartesian coordinates in the pre-defined working range are highly nonlinear, described in section II. Corresponding control strategies are studied according to these challenging properties of the new magnetic topology.

Many control strategies dealing with the high nonlinearity of the unstable electromagnetic actuators were studied in previous researches. One popular method was to derive a nominal linear model by linearizing the system at one working-point and modeling the nonlinearities as perturbations. Subsequently, a robust controller design method, such as $\mu$-synthesis [6,7] or $H_{\infty}$ [2,8] was employed. However, the high nonlinearity of an electromagnetic actuator led to large perturbations, which created conflicts between robust performance and robust stability. Feedback linearization [5] was another option to compensate the highly-nonlinear permanent magnetic force.

Control strategies which simultaneously achieve stabilization and vibration isolation of a non-contact electromagnetic actuator were also studied by other researchers. Stabilization and vibration isolation was achieved in [1] by absolute position feedback, which is not feasible in this application. A 6-DOF suspension system composed of four non-contact electromagnetic actuators was 
built [4] for a payload of maximal 200 [kg]. A PI controller was used to stabilize the electromagnetic actuator based on relative position feedback. An $H_{\infty}$ controller was used to isolate the floor vibration based on absolute acceleration feedback and it also took care of the changing parameters and uncertainties simultaneously. However, to stabilize the electromagnetic actuator with such high nonlinearity to guarantee robust vibration isolation performance by only a PI controller is very difficult.

This paper focuses on the control of a single electromagnetic actuator with three translational DOF rather than the magnetic topology design. The properties of the electromagnetic actuator were analyzed. A control strategy combining feedback linearization and $H_{\infty}$ control was applied to the electromagnetic actuator model which was built in Matlab for real-time simulation before manufacturing. The relative position (between translator and the stator) signals and the high-pass filtered absolute acceleration were used for feedback control. The performance of vibration isolation in a working range where the plant shows high nonlinearity was evaluated by simulation.

\section{MOdEl ANAlysis OF THE ACtUAtor}

The electromagnetic actuator is composed of permanent magnets and coils. It can be divided by functionality into two main components: a translator and a stator. There is no mechanical contact between the translator and the stator. Both the translator and the stator are integrated with permanent magnets, which provide passive force mainly in the vertical direction (z-axis). The active force is purely based on the Lorentz force exerted on coils so that it has linear relation with the current.

In the final application, three electromagnetic actuators will be used to replace the three air bearings shown in Fig. 1(b) to support a single rigid payload. Since the electromagnetic actuators are designed for a very short stroke (in scales of millimeters) and the distances between them are all larger than one meter, the rotational displacements of the payload would be very small. Therefore, the translator rotations are not included in this study. The mass center of the translator and payload is assumed to coincide with the point where the magnetic forces are applied and it is also the position where the relative displacement sensor is installed. This position is also set as the origin of the Cartesian coordinate system.

The model of an electromagnetic actuator has six inputs: three currents $\left(I_{i}\right)$ and three floor displacements $\left(d_{G i}\right)$; and six outputs: three absolute displacements of the translator $\left(d_{A i}\right)$ and three relative displacements of the translator with respect to the stator $\left(d_{R i}\right)$. The subscript $i=x, y, z$ denote the three Cartesian axes. The payload and translator mass is denoted by $M$ and the gravitational acceleration is denoted by $g$. The relations between the absolute displacements and the relative displacements are

$$
d_{A i}=d_{R i}+d_{G i} \quad \forall i \in\{x, y, z\} .
$$

A 2-D representation of the electromagnetic actuator under vibration is shown in Fig. 2. Note that this figure does not indicate the physical design of the magnetic topology.
The stator is rigidly fixed on the floor where the vibrations come from and the translator is rigidly fixed to the payload (not shown in this figure). The dashed square in the middle of stator shows the initial position (the equilibrium point) of the translator. The coordinate system for $d_{R i}$ is fixed to the stator. The working range is defined as the cubic space $\left|d_{R i}\right| \leq$ $l[\mathrm{~mm}]$. The equations of motion of the translator are:

$$
M \ddot{d}_{A i}=F_{p i}+F_{a i}-F_{C i} \quad \forall i \in\{x, y, z\} .
$$

where $F_{p i}$ is the passive force generated by permanent magnets and $F_{a i}$ is the active force generated by the actuator. $F_{C i}$ denote the static forces: $F_{C x}=F_{C y}=0, F_{C z}=\mathrm{Mg}$. The active force $F_{a i}$ has linear and decoupled relation with the corresponding coil current:

$$
F_{a i}=\mu_{i} I_{i} \quad \forall i \in\{x, y, z\} .
$$

The magnetic force $F_{p i}$, shown in Fig. 3-Fig. 6, is highly dependent on the $d_{R i}$. This also holds for the coupling among the three DOF. $F_{p i}$ have six symmetry properties:

$$
\begin{aligned}
& \text { - } F_{p x}\left(d_{R x}, d_{R y}, d_{R z}\right)=F_{p x}\left(-d_{R x}, d_{R y}, d_{R z}\right) \\
& \text { - } F_{p x}\left(d_{R x}, d_{R y}, d_{R z}\right)=-F_{p x}\left(d_{R x},-d_{R y}, d_{R z}\right) \\
& \text { - } F_{p x}\left(d_{R x}, d_{R y}, d_{R z}\right)=F_{p y}\left(d_{R y}, d_{R x}, d_{R z}\right) \\
& \text { - } F_{p z}\left(d_{R x}, d_{R y}, d_{R z}\right)=F_{p z}\left(-d_{R x}, d_{R y}, d_{R z}\right) \\
& \text { - } F_{p z}\left(d_{R x}, d_{R y}, d_{R z}\right)=F_{p z}\left(d_{R x},-d_{R y}, d_{R z}\right) \\
& \text { - } F_{p z}\left(d_{R x}, d_{R y}, z^{*}+d_{R z}\right)=F_{p z}\left(d_{R x}, d_{R y}, z^{*}-d_{R z}\right)
\end{aligned}
$$

where $\mathrm{z}^{*}=-0.2[\mathrm{~mm}]$. Shown in Fig. 5, $z_{0}:\left(d_{R x}, d_{R y}, d_{R z}\right)=(0$, $\left.0, z^{*}\right) \quad$ has an important property. All entries in the $3 \times 3$ stiffness matrix $\mathrm{K}$ defined by

$$
K\left(d_{R x}, d_{R y}, d_{R z}\right)=-\frac{\partial F_{p i}}{\partial d_{R j}} \quad \forall i, j \in\{x, y, z\},
$$

are zero. If the translator moves up to a position $\left(0,0, z^{*}+c\right)$, $c>0$, the actuator is stable along vertical DOF but unstable at the two horizontal DOF's. If the translator moves down to a position $\left(0,0, z^{*}-c\right), c>0$, the actuator is unstable along vertical DOF but is stable at the two horizontal DOF's. This stability change phenomenon increases the difficulty of the controller design. The equilibrium point is selected to be close to the zero-stiffness point because it is practically not possible to make the payload gravity exactly the same as the vertical permanent magnetic force at $z_{0}$.

\section{CONTROL STRATERGY}

The control objective is to simultaneously fulfill the following requirements within the predefined working range.

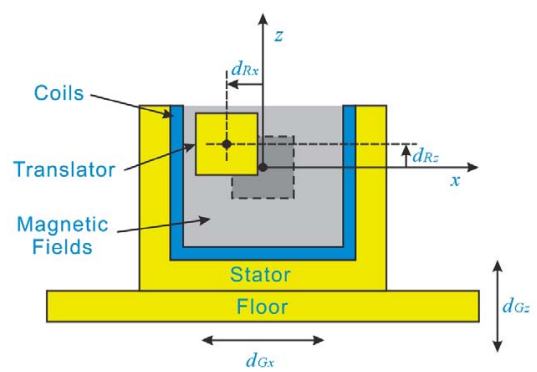

Figure 2. 2D Representation of the Electromagnetic Actuator 


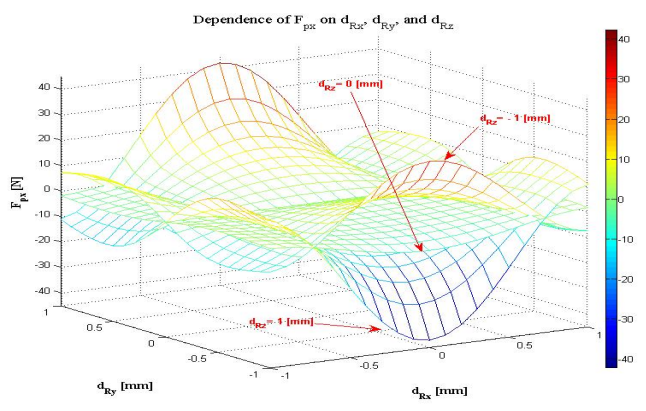

Figure 3. Permanent magnetic force $F_{p x}$ with $d_{R z}=0,-1,1[\mathrm{~mm}]$

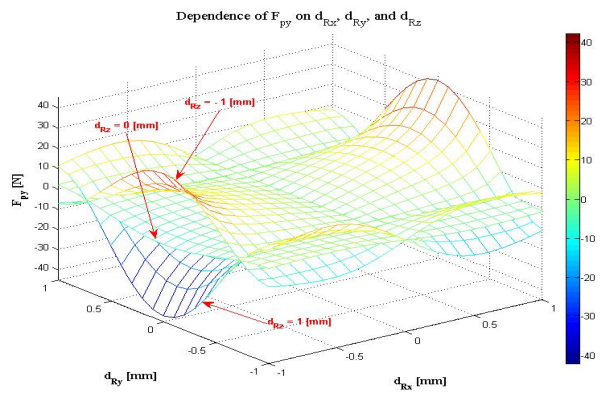

Figure 4. Permanent magnetic force $F_{p y}$ with $d_{R z}=0,-1,1[\mathrm{~mm}]$

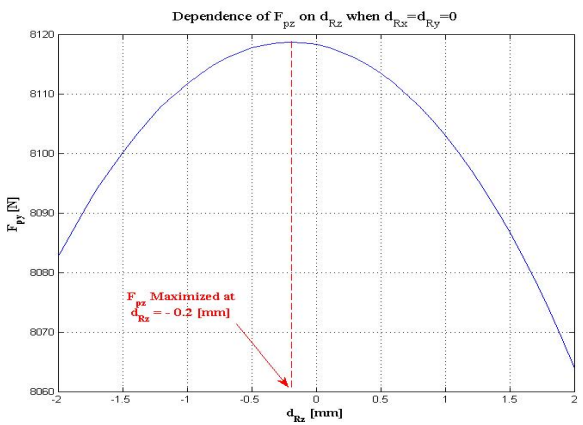

Figure 5. Permanent magnetic force $F_{p z}$ with $d_{R x}=d_{R y}=0$

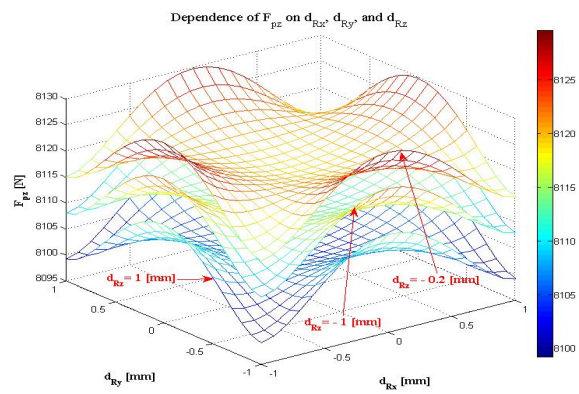

Figure 6. Permanent magnetic force Fpz with $\mathrm{dRz}=0,-1,1[\mathrm{~mm}]$

- To stabilize the highly nonlinear 3-DOF plant (electromagnetic actuator with payload).

- To reduce the mid and high frequency vibration transmitted from floor to the payload.
- To reject the disturbance force directly applied on the payload.

- To track the low frequency reference change with relaxed settling time requirement.

In the final application, the permanent magnetic force applied on translator is time-invariant. Therefore, it can be measured at selected positions by a force sensor. Then, a lookup table formed by the measured data or a formula calculated by curve fitting of the measured data can be used to calculate the permanent magnetic force in real time. Note that the static force $F_{C i}$ is measured together with $F_{p i}$. The force calculation error, the difference between the calculated force and the real permanent magnetic force, is expected to be small and slow-varying. It can be modeled as force disturbance and solved by an active controller. Denote the calculated passive magnetic force and the static force by $F_{p i}$ ' and $F_{C i}{ }^{\prime}$, respectively. The active force $F_{a i}$ can be written as

$$
F_{a i}=-F_{p i}^{\prime}+F_{C i}^{\prime}+u_{i}, \quad \forall i \in\{x, y, z\},
$$

where $u_{i}$ is the desired control force for the linearized model. With (5), the equations of motion (2) can be modified to

$$
M \ddot{d}_{A i}=u_{i}+E_{i}, \quad \forall i \in\{x, y, z\} .
$$

where $E_{i}=F_{p i}+F_{C i}{ }^{\prime}-F_{p i}{ }^{\prime}-F_{p i}$ is the force calculation error. Using feedback linearization, not only the highly nonlinear magnetic forces are compensated, but also the Multi-Input Multi-Output (MIMO) controller design problem is decoupled to three Single-Input Single-Output (SISO) controller design problems. The plant model for each SISO system is reduced to a disturbed double integrator, which is defined as the nominal model. Since the controller design strategy for each SISO system is exactly the same, only the design strategy for the vertical DOF ( $z$-axis) is described. The state vector of the nominal model $P$ is denoted by $x_{m}$.

The overall control diagram is shown in Fig. 7. The high nonlinearity is compensated by feedback linearization. The signal $a_{A i}$ is the absolute acceleration of the translator. The high-pass filter is used to simulate the sensitivity drop of the industrial acceleration sensors at low frequencies.

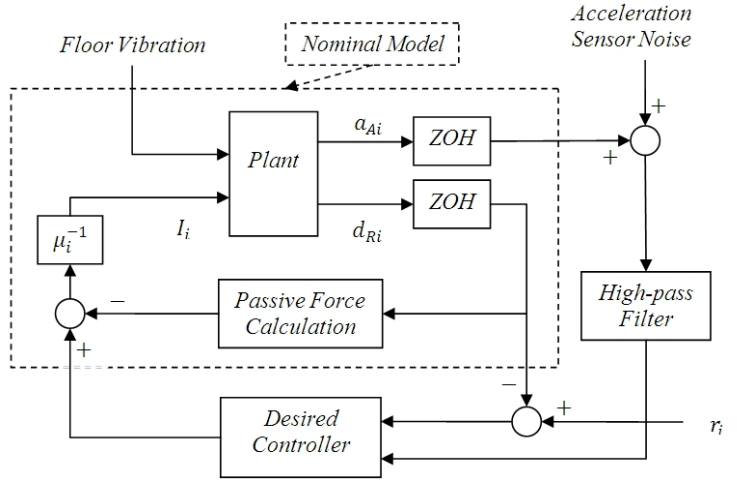

Figure 7. Control diagram of feedback linearization. 


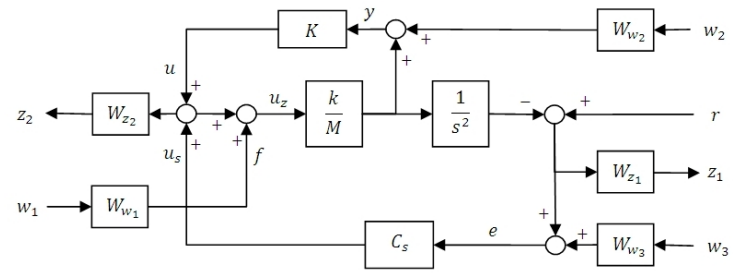

Figure 8. Control diagram for the nominal model.

The desired controller is designed to fulfill the above requirements for the nominal model. It is composed of a stabilizing controller $C_{s}$ and an $H_{\infty}$ controller $K$. The stabilizing controller $C_{s}$ is designed with the absence of $K$. The $H_{\infty}$ controller is designed based on the control diagram shown in Fig. 8. The design objective of $C_{s}$ is to stabilize the nominal model and at the same time, the tracking error $e$ in Fig. 8 should be zero under DC component in $w_{l}$. This property makes $C_{s}$ a candidate controller to measure the DC component of the force calculation error. A good performance of vibration isolation requires that the loop gain $C_{s} P$ has a low bandwidth and a low gain. The state vector of $C_{s}$ is denoted by $x_{s}$. Denoting the states of the weights $W_{w 1}$, $W_{w 2}, W_{w 3}, W_{z 1}, W_{z 2}$ by $x_{w 1}, x_{w 2}, x_{w 3}, x_{z 1}, x_{z 2}$, respectively, the generalized plant $P$ can be described by

$$
\left\{\begin{array}{l}
\dot{x}=A x+B_{1} w+B_{2} u, \\
z=C_{1} x+D_{11} w+D_{12} u, \\
y=C_{2} x+D_{21} w+D_{12} u,
\end{array}\right.
$$

where $x=\left[x_{m}, x_{s}, x_{w 1}, x_{z 1}, x_{z 2}, x_{w 2}, x_{w 3}\right]^{T}, w=\left[w_{1}, w_{2}, w_{3}\right]^{T}$, $z=\left[z_{1}, z_{2}\right]^{T}, y$ is the measured acceleration and $K$ is the $H_{\infty}$ controller as in Fig. 8.

The other signals and blocks in Fig. 8 are described as follows. The input $r$ is the reference signal. In the block $k / M$, $M$ is the payload mass and $k$ is a constant to change the output unit: $k=1$ for [m] and $k=1000$ for [mm]. The input $w_{1}$ is the sum of the force calculation error and the force disturbances. The amplitude decreases with rising frequency. Therefore, $W_{w 1}$ was designed to be a low-pass filter. The input $w_{2}$ represents the acceleration sensor noise. Based on experience, the industrial acceleration sensors have either relatively very high noise or sharp gain drop at low frequencies but the noise is very low at high frequencies. Therefore, $W_{w 2}$ was designed as a second-order low-pass filter. The floor vibration is denoted by $w_{3}$ and the amplitude is similar for all frequencies. However, the design objective is to follow the low frequency vibration and to isolate the high frequency vibration. Therefore, $W_{w 3}$ was designed as a high-pass filter. The amplitude of the payload displacement $z_{1}$ should decrease as fast as possible with the rising frequency. So $W_{z 1}$ was designed as a high-pass filter. The output $z_{2}$ represent the total control effort. Its amplitude is limited by the actuator capability of how fast the control signal could be followed. Since this capability drops at high frequencies, $W_{z 2}$ was designed as a high-pass filter so that $z_{2}$ is suppressed at high frequencies.

\section{PERformance Simulation}

A Matlab model for the new 3-DOF, highly nonlinear electromagnetic actuator is built for real-time simulation. The mass of the payload and the translator is $M=827.55[\mathrm{~kg}]$. Force-position relations $F_{p i}$ are derived by least-square curve fitting of the force-position data, which is calculated based on the magnetic topology [3]. The default units for displacement and force are $[\mathrm{mm}]$ and $[\mathrm{N}]$, respectively. The designed stabilizing controller is

$$
C_{s}=\frac{40000(s+0.3474)(s+0.05756)}{s(s+4)} .
$$

The weights are designed as

$$
\begin{gathered}
W_{w 1}=\frac{300}{s+150}, W_{w 2}=\frac{(s+3)^{2}}{(s+0.001)^{2}}, W_{z 2}=\frac{0.01(s+6)}{s+5000}, \\
W_{w 3}=\frac{6(s+0.0004)}{s+50}, W_{z 1}=\frac{11000(s+0.5)}{s+5500} .
\end{gathered}
$$

The $H_{\infty}$ controller is calculated in Matlab by 'hinfsyn'. Both the $H_{\infty}$ controller and the stabilizing controller $C_{s}$ are converted to $Z$-domain by the zero-pole match method.

The simulation was carried out to simulate the real conditions in the final application. The floor vibration $w_{3}$ was simulated by the white noise and most of this noise signals were assumed to be within $\pm 0.1[\mathrm{~mm}]$. The acceleration sensor noise $w_{2}$ was simulated by a low-pass filtered white noise because the industrial acceleration sensors had relatively large noise at low frequencies and relatively low noise at high frequencies. The acceleration noise signal is assumed to be within $\pm 3 \%$ of the acceleration signal. The slow-varying force calculation error $E_{i}$ is assumed to be

$$
E_{i}=-\left(1+4 d_{R i}\right), \quad \forall i \in\{x, y, z\} .
$$

The force disturbance directly applied on the payload was simulated by white noise. Therefore, $w_{1}$ for each DOF was simulated by the sum of $E_{i}$ and white noise. This white noise is assumed to be within $\pm 1[\mathrm{~N}]$. The sampling frequency for all simulations was $10[\mathrm{kHz}]$.

Under floor vibration $w_{3}$ and the total force disturbance $w_{1}$, the reference following performance, shown in Fig. 9(a), was simulated by tracking a low-pass filtered step signal. Note that the low-pass filtered step signal is used as the reference to avoid the oscillations, which is feasible in the final application. The absolute displacement in Fig. 9(a) stabilized at the target position with approximately zero steady-state error. The step responses for the three DOF are almost the same because both the nonlinearity and coupling are removed by feedback linearization.

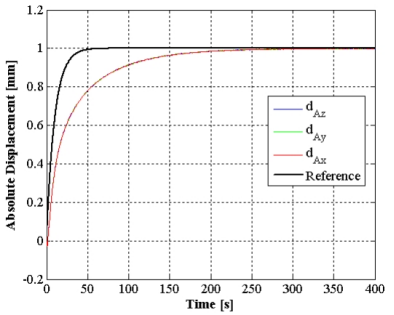

(a) Reference-following performance

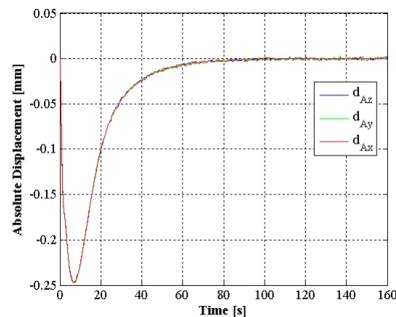

(b) Response to force disturbance
Figure 9. Time domain response. 


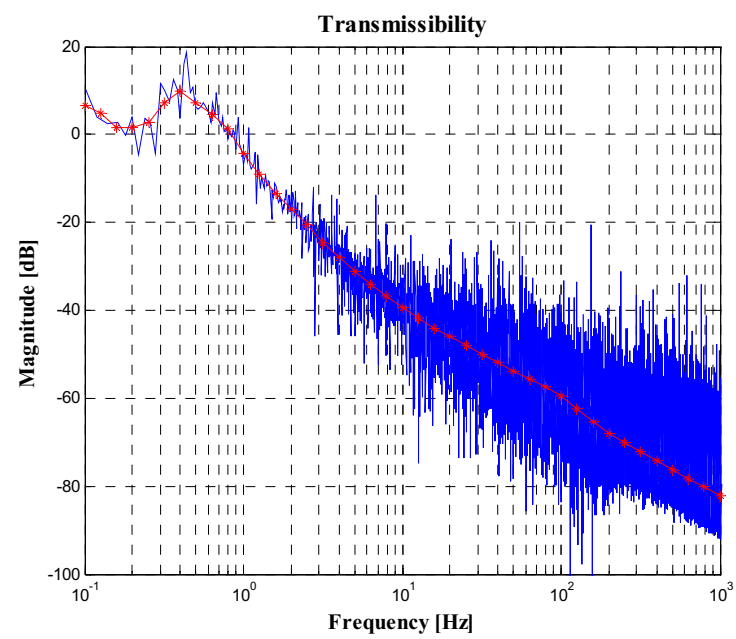

Figure 10. Transmissibility at working range boundary.

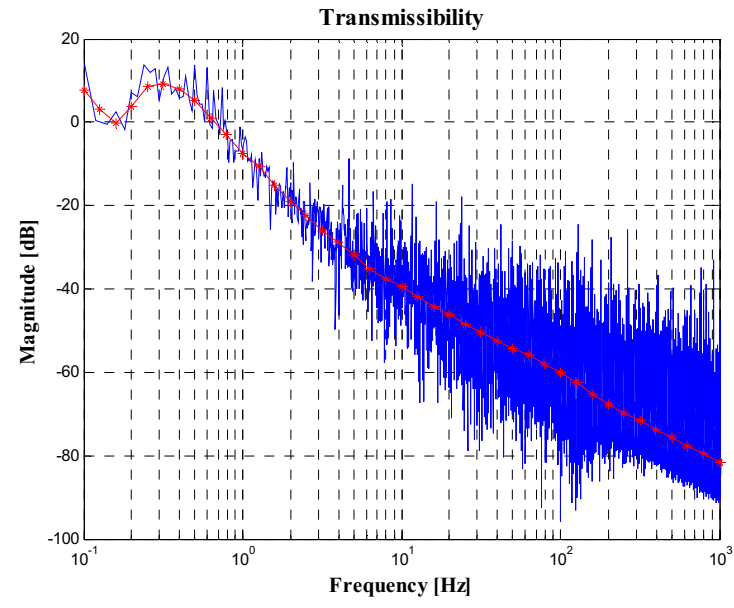

Figure 11. Transmissibility at the origin.

In the step response for the z-axis, the signal $d_{A z}$ and floor vibration $d_{G z}$ in the last $50[\mathrm{~s}]$ is used to calculate the magnitude of the transmissibility, which is defined by the transfer from the floor displacement to the payload absolute displacement, shown in Fig. 10. The blue curve in Fig. 10 is the transmissibility magnitude directly calculated by the Discrete Fourier Transform (DFT) of the absolute displacement over the DFT of the floor displacement. Since the floor displacement was simulated by the white noise generated by Matlab, its amplitudes have increasing deviations with frequencies. This deviation leads to large noise at high frequencies in the magnitude plot of transmissibility. A smoothing algorithm, which averages the neighbor frequency amplitudes for chosen frequencies, was applied to both $d_{G z}$ and $d_{A z}$. Based on the smoothed signals, the transmissibility magnitude was calculated and plotted as the curve marked by red stars in Fig. 10. Under floor vibration $w_{3}$, the response of translator absolute displacement to suddenly applied total force disturbance $w_{1}$ is plotted in Fig. 9(b). It shows that the steady-state error is also approximately zero. It also shows that the DC component of force calculation error would be reduced to a level much smaller than $1[\mathrm{~N}]$ if the passive magnetic force is measured by the proposed controller. The signals of $d_{G z}$ and $d_{A z}$ in the last 50 [s] are used to plot the magnitude of the transmissibility, shown in Fig. 11. Same as Fig. 10, both the raw and the smoothed transmissibility magnitude curves are plotted. Comparing Fig. 10 with Fig. 11, it can be concluded that the vibration isolation performance is robust within the predefined working range with the proposed control strategy. The transmissibility magnitudes for the horizontal DOF are not shown because the corresponding curves are almost the same as Fig. 10 and Fig. 11.

\section{CONCLUSIONS}

The proposed control strategy of combining feedback linearization and $H_{\infty}$ control was applied to the model of a recently designed 3-DOF electromagnetic actuator with large load. This actuator is inherently unstable and the passive characteristics are highly nonlinear. Simulation results show that the step response has approximately zero steady-state error under floor vibration and slow-varying force calculation error. The steady-state error in the response to the force disturbance with DC components is also zero. Robust vibration isolation performance was achieved within a working range of high nonlinearity.

\section{ACKNOWLEDGMENT}

This work is within the Gaussmount Project, which is a part of the Dutch IOP-EMVT program and is supported financially by SenterNovem, an agency of the Dutch Ministry of Economic Affairs.

\section{REFERENCES}

[1] Kosuke Nagaya and Masashi Ishikawa, "A Noncontact Permanent Magnet Levitation Table with Electromagnetic Control and Its Vibration Isolation Method Using Direct Disturbance Cancellation Combining Optimal Regulators", IEEE Trans. on Magnetics, vol. 31, No. 1. January 1995.

[2] Ken'inchi Nakashima, Taro Tsujino and Takao Fujii, "Multivariable Control of a Magnetic Livitation System Using Closed Loop Identification and \$H linfty\$ Control Theory", Proc. 35th Conf. on Decision and Control, Kobe, Japan, December 1996.

[3] J.L.G. Janssen, J.J.H. Paulides, E. Lomonova, "Passive Limitations For a Magnetic Gravity Compensator", Journal of System Design and Dynamics, vol. 3, No. 4. p.671-680. July 2009.

[4] Katsuhide WATANABE, Shinji HARA, Yoichi KANEMITSU, Takahide HAGA, Kenichi YANO, Takayuki MIZUNO, Ryuta KATAMURA, "Combination of $\mathrm{H} \infty$ and PI Control for an Electromagnetically Levitated Vibration Isolation System", Proc. 35th Conf. on Decision and Control, Kobe, Japan, December 1996.

[5] H. Nijmeijer and A. J. van der Schaft, Nonlinear Dynamical Control Systems, Springer, 1990.

[6] J.L. Lin and B.C. Tho, Analysis and $\mu$-Based Controller Design for an Electromagnetic Suspension System, IEEE Trans. on Education, vol. 41, No. 2, May 1998.

[7] M. Fujita, T. Namerikawa, F. Matsumura, and K. Uchida, $\mu$-Synthesis of an Electromagnetic Suspension System, IEEE Trans. on Automatic Control, vol. 40, No. 3, March 1995.

[8] T. Namerikawa and M. Fujita, " Hळ DIA Control of Magnetic Suspension Systems", Proc. 2004 IEEE Intemational Conf. on Control Applications, Taipei, Taiwan, September 2-4, 2004. 